\title{
Evolution-based uncertainty design for artificial systems
}

\author{
Boqiang Shi ${ }^{1, a}$, Yanhua Shen ${ }^{1, b^{*}}$ \\ ${ }^{1}$ School of Mechanical Engineering, University of Science and Technology Beijing, \\ Beijing 100083, China \\ ashiboqiangustb@163.com, byanhua_shen@ces.ustb.edu.cn
}

\begin{abstract}
Keywords: Evolution-Based Uncertainty Design (EBUD); Reliability Design; Ito Lemma; Artificial System

Abstract. The effect of uncertainty and its evolution with time on the system reliability design are investigated in this paper. The system design variables are modeled in Geometric Brownian Motions which using the drift rate and diffusion rate to describe the evolution-based uncertainty of variables. Based on Ito Lemma, the system performance composed with these variables can also be expressed with drift function and diffusion function. Using mathematic transformation for the time series data of each variables, the drift function and diffusion function of the system can be calculated to reflect the system performance evolution uncertainty characteristic. The system evolution-based uncertainty reliability is defined as the probability function of system performance output and allowable performance output. Evolution-based uncertainty design method is developed in which the system can be designed with the specified reliability value at the given time period. The example results show the effectiveness of the proposed method on dealing with the system evolution-based uncertainty.
\end{abstract}

\section{Introduction}

In real world, human activities have involved the land, deep sea and extended to the outer space. A lot of artificial systems, such as nuclear power plant, offshore drilling platform, spacecraft and space station, have been invented to expand the scope of these human activities. Generally, these artificial systems are very complicated and possess multiple variables. The nonlinearity, uncertainty of the variables are mainly the characteristic of the complex system. The reliability problem of such artificial system is always concerned both in the engineering and academic research field.

At present, the reliability design method is based on the probability theory in which the system variables (or parameters) are regarded as stochastic variable [1]. There exists two major problems: one is we need a large amount of data to determine the distribution and numerical characteristics of stochastic variables. Secondly, the reliability of system in this method doesn't consider the system evolution-based uncertain factor [2].

Many research works have been proposed for the time-dependent reliability analysis of the system. Monte Carlo Simulation (MCS) can be used for time-dependent reliability analysis, but it is computationally expensive. Method such as the upcrossing rate is the most widely used one [3 5]. However, it is not easy to obtain the upcrossing rate, some assumption or effective way is made to improve this method. Du has developed a more accurate method by combining the joint upcrossing rate with First Order Reliability Method (FORM) in literature [6]. Even so, there exist a lot of error sources in this method for accurately estimating the time-dependent reliability of system.

In order to analyze the evolution-based uncertain factors on the system, Shi uses Ito process to describe the machine parts stress and strength, and proposed a method to calculate the mechanical system reliability incorporating time variation and uncertainty $[7,8]$, but he didn't use it for designing the system in the view of evolution-based uncertainty.

At present, there is no related research work about how to design the system based on the evolution uncertain reliability. Unlike the traditional reliability design method, this paper proposes an evolution-based uncertain design (EBUD) method to investigate the uncertainty and time-dependent characteristic of the system. This method can forecast the reliability of artificial system at any time based on drift and diffusion characteristic of the basic design parameters. 


\section{Ito Process and Ito Lemma}

For a process $x_{t}$ whose state changes continuously with time, it can be described as Ito process:

$$
d x_{t}=\mu\left(x_{t}, t\right) d t+\sigma\left(x_{t}, t\right) d w_{t}
$$

where, $w_{t}$ is a Wiener process (Brownian motion), $\mu\left(x_{t}, \mathrm{t}\right)$ drift function and $\sigma\left(x_{t}, \mathrm{t}\right)$ diffusion function. $\mu\left(x_{t}, \mathrm{t}\right)$ and $\sigma\left(x_{t}, \mathrm{t}\right)$ are deterministic functions depending on $x_{t}$ and time $\mathrm{t}$ respectively. Different stochastic process will be generated if expressions of the drift function and diffusion function are different.

If we know the stochastic process followed by $x$, Ito Lemma tells us the stochastic process followed by some function $G(x, t)$. According to the derivative chain rule of random variables and stochastic calculus, Ito Lemma states that $G(x, t)$ follows the Ito process:

$$
\mathrm{d} G=\left(\frac{\partial G}{\partial x} \mu+\frac{\partial G}{\partial t}+\frac{1}{2} \frac{\partial^{2} G}{\partial x^{2}} \sigma^{2}\right) \mathrm{d} t+\frac{\partial G}{\partial x} \sigma \mathrm{d} w_{t}
$$

where, drift function $\frac{\partial G}{\partial x} \mu+\frac{\partial G}{\partial t}+\frac{1}{2} \frac{\partial^{2} G}{\partial x^{2}} \sigma^{2}$, and diffusion function $\frac{\partial G}{\partial x} \sigma$.

Given a set of Ito processes $x_{i}(i=1, \ldots \ldots, \mathrm{n})$ :

$$
\mathrm{d} x_{i}(t)=\mu_{i}\left(x_{t}, d t\right) \mathrm{d} t+\sigma_{i r}\left(x_{t}, t\right) \mathrm{d} w_{r}, \quad i=1,2, \mathrm{~L}, n ; r=1,2, \mathrm{~L}, m
$$

where, $\mu_{i}\left(x_{t}, d t\right)$ is drift function, and $\sigma_{i r}\left(x_{t}, t\right)$ is a $\mathrm{n} \times \mathrm{m}$ correlated diffusion matrix.

Using the Taylor's series expansion of $G(x, t)$ ( ignoring the terms of higher order than $\mathrm{d} t$ ), the multidimensional Ito Lemma can be written as follows :

$$
\mathrm{d} G=\left[\frac{\partial G}{\partial t}+\sum_{i}^{n} \frac{\partial G}{\partial x_{i}} \mu_{i}+\frac{1}{2} \sum_{i}^{n} \sum_{j}^{n} \frac{\partial^{2} G}{\partial x_{i} \partial x_{j}}\left(\sigma \sigma^{T}\right)_{i j}\right] \mathrm{d} t+\sum_{i=1}^{n} \frac{\partial G}{\partial x_{i}} \sigma_{i r} \mathrm{~d} w_{r}
$$

where, drift function $\frac{\partial G}{\partial t}+\sum_{i}^{n} \frac{\partial G}{\partial x_{i}} \mu_{i}+\frac{1}{2} \sum_{i}^{n} \sum_{j}^{n} \frac{\partial^{2} G}{\partial x_{i} \partial x_{j}}\left(\sigma \sigma^{T}\right)_{i j}$, and diffusion function $\sum_{i=1}^{n} \frac{\partial G}{\partial x_{i}} \sigma_{i r}$.

\section{General Principle of Evolution-Based Uncertainty Design (EBUD)}

In order to analyze the system evolution characteristic quantitatively, the system status can be described by its basic parameters, and it also can be the variables which are used in the system's kinematic and dynamic equation. The status of artificial system is described by the system functional output or additional output, all of these is named as performance output of the system.

\section{Definition}

Performance output $F_{\text {real }}(X, t)$ : a status expression of artificial system $S$ at any time $t$. It is a multivariate function of variables $X$ and time $t$, where the variables $X=\left[x_{i}(t)\right]^{\mathrm{T}}(i=1,2 \ldots \ldots \mathrm{n})$, and $x_{i}(t)$ is an Ito process expressed in equation (1). For simplicity, $F_{\text {real }}(X, t)$ is represented as $F(t)$. Performance output $F(t)$ of the system can be observable, and it may be a composite function composed of multi-observable physical quantities.

Allowable performance output $F_{\text {allow }}(Y, t)$ : it is also a multivariate function of variables $Y$ and time $t$, where $Y=\left[y_{i}(t)\right]^{\mathrm{T}}(i=1,2 \ldots \ldots \mathrm{m})$, and $y_{i}(t)$ is an Ito process expressed in equation (1). For simplicity, $F_{\text {allow }}(Y, t)$ is represented as $[F(t)]$, which is an expression of performance (or status) requirement of system $S$ at any time $t$. Allowable performance output can be some performance index of system $S$ with actual physical significance. It also can be unobservable, abstract function composed of several observable physical quantities.

Based on the above definitions, the performance (status) output $F(t)$ and allowable performance output $[F(t)]$ of the system will be time-dependent function which is composed of design variables. Followings are some definitions of EBUD:

EBUD focuses on the time-varying, evolution and uncertainty characteristics existing in the artificial system $S$. The quantification expression of the system uncertain or risky can be described by the 
uncertainty/un-reliability, or by certainty/ reliability. Uncertainty and reliability of the system in the EBUD will be calculated with performance output and allowable performance output which are expressed by Ito process.

\section{Design guideline for EBUD}

For an artificial system, the state variable of the system can be considered as continuous stochastic processes. The system uncertainty at any time $t$ will be resolved by the probability model which is determined by the relationship of actual performance output 'value' and the required 'value'.

The math expression of the system meeting the performance output $F(t)$ is the inequality relationship between the performance output and allowable performance output. Its general expression can be described as follows:

$$
F(t) \leq[F(t)]
$$

If the system requires the performance output $F(t)$ greater than or equal to the allowable performance output, equation (5) can be handled as this:

$$
\left\{\begin{array}{l}
F^{\prime}(t)=-F(t) \\
{\left[F^{\prime}(t)\right]=-[F(t)]}
\end{array}\right.
$$

According to the above definition, the system reliability over a time interval $\left[t_{0}, t_{s}\right]$ for the evolution-based uncertainty analysis can be written as:

$$
P\left(t_{0}, t_{s}\right)=P\left\{F(t) \leq[F(t)], \forall t \in\left[t_{0}, t_{s}\right]\right\}
$$

For the performance output $F(t)$ and allowable performance output $[F(t)]$ can be expressed with Ito process, the reliability $P\left(t_{0}, t_{s}\right)$ in equation (7) is a time-variant function.

\section{Drift function and diffusion function of system}

In the model of EBUD, the fluctuation of system is expressed with drift function. Drift function is used to describe the system's variation. The uncertain characteristic of the system is expressed with diffusion function. It is used to describe the uncertainty of system behavior at any time.

If the system $S$ is composed of many subsystems, the performance output $F(t)$ of the system will be composite functions of many variables which belong to these subsystems. According to abovementioned Ito Lemma, drift function $\mu\left(x_{t}, t\right)$ and diffusion function $\sigma\left(x_{t}, t\right)$ of performance output $F(t)$ can be given by the subsystems' drift function and diffusion function.

I Drift function and diffusion function of $\ln F(t)$

If a variable follows a standard Weiner process, it has drift rate 0 and variance rate 1 . For a generalized Wiener process, the variable $x_{t}$ is a generalized Wiener process with the following form:

$$
\mathrm{d} x_{t}=\mu \mathrm{d} t+\sigma \mathrm{d} w_{t}
$$

where, $\mu$ and $\sigma$ are some certain values, $t$ is continuous time.

Thus, based on Ito Lemma and literature [1], we can prove that:

$$
E\left(x_{t}-x_{0}\right)=\mu t, \operatorname{Var}\left(x_{t}-x_{0}\right)=\sigma^{2} t
$$

The results in equation (9) demonstrate that the $x_{t}-x_{0}$ in time interval $[0, t]$ will follow the normal distribution $\mathrm{N}\left(\mu t, \sigma^{2} t\right)$.

For an artificial system with performance output $F(t)$ ( an Ito process with the drift function $\mu_{F}$, the diffusion function $\sigma_{F}$ ), if we set transformation $G(F(t), t)=\ln F(t)$, according to Ito Lemma in equation (2), it can lead to:

$$
\begin{aligned}
\mathrm{d} \ln F(t) & =\left(\frac{1}{F(t)} \cdot \mu_{F} \cdot F(t)-\frac{1}{2} \frac{1}{F^{2}(t)} \cdot \sigma_{F}{ }^{2} F^{2}(t)\right) \mathrm{d} t+\frac{1}{F(t)} \cdot \sigma_{F} \cdot F(t) \mathrm{d} w_{t} \\
& =\left(\mu_{F}-\frac{\sigma_{F}^{2}}{2}\right) \mathrm{d} t+\sigma_{F} \mathrm{~d} w_{t}
\end{aligned}
$$

The equation (10) indicates that $\ln \mathrm{F}(\mathrm{t})$ is a generalized Weiner process which follows a normal 
distribution with mean $\hat{\mu}_{\ln F}(t)=\ln F(0)+\left(\mu_{F}-\frac{\sigma_{F}^{2}}{2}\right) t$, variance $\hat{\sigma}_{\ln F}^{2}(t)=\sigma_{F}^{2} t$ at time $t$. And it also means that the $\ln F(t)$ is an Ito process with drift function $\mu_{F}-\sigma_{F}^{2} / 2$, diffusion function $\sigma_{F}$.

\section{System reliability in EBUD}

As abovementioned, if the performance output $F(t)$ of the system following the Ito process, according to equation (10), $\ln F(t)$ will follow a normal distribution. It is the same for the allowable performance output $[F(t)]$, that is $\ln [F(t)]$ will also follow a normal distribution.

In practical engineering application, in order to easily calculate the above reliability from the view point of EBUD, each item in the relationship (7) is taken logarithm simultaneously:

$$
P\{\ln F(t) \leq \ln [F(t)]\}(F(t)>0)
$$

or

$$
P\left\{\ln F^{\prime}(t) \leq \ln \left[F^{\prime}(t)\right]\right\}\left(F^{\prime}(t)>0\right)
$$

In EBUD, the system $S$ with allowable performance output $\ln [F(t)]$ is designed which it should satisfy the equation (11) or (12).According to equation (11), the reliability of system varies with time $t$. It is defined by the probability that an Ito process $\ln F(t)$ is lower than another Ito process $\ln [F(t)]$.

As shown in Fig. 1, the situation of $\ln F(t)$ larger than $\ln [F(t)]$ represents the system at this moment will be considered as unacceptable or having risk. Once the distribution and parameters of $\ln F(t)$ and $\ln [F(t)]$ are determined, the reliability $\mathrm{R}[t]$ can be calculated by estimating the probability $X>Y$ as following:

$$
R(t)=\int_{-\infty}^{\infty} \int_{Y}^{\infty} f(Y) f(X) d X d Y
$$

where, $Y=\ln [F(t)], f(Y)$ is the probability density function (pdf) of allowable performance output $\ln [F$ $(t)]$, and $X=\ln F(t), f(X)$ is the probability density function (pdf) of performance output $\ln F(t)$.

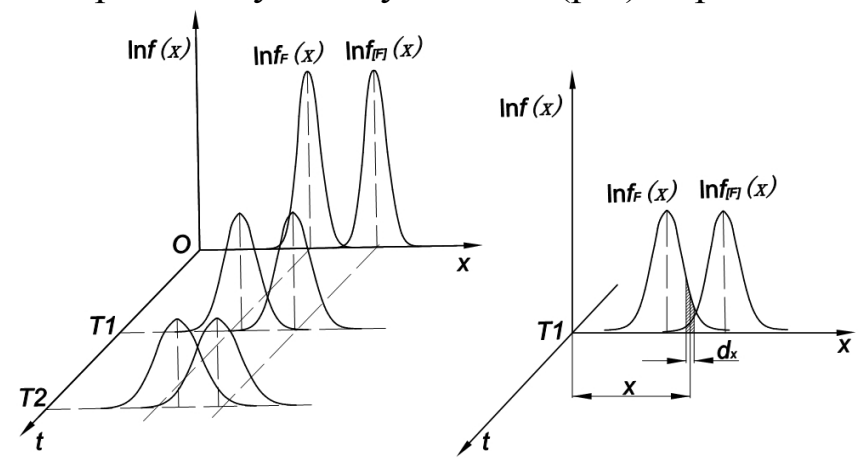

Fig.1 Evolution of interference model of system reliability

However, it is difficult to obtain the probability density function of $X=\ln F(t), Y=\ln [F(t)]$ at any time. As we know that $\ln F(t), \ln [F(t)]$ follow normal distribution, the system reliability $\mathrm{R}(t)$ during time interval $\left[t_{0}, t_{s}\right]$ can be given:

$$
\begin{aligned}
R(t) & =\mathrm{P}\left\{\ln F(t) \leq \ln [F(t)], \forall t \in\left[t_{0}, t_{s}\right]\right\} \\
& =\Phi(\beta(t))=\Phi\left(\frac{\hat{\mu_{\ln [F]}(t)-\hat{\mu}_{\ln F}(t)}}{\sqrt{\hat{\sigma}_{\ln [F]}^{2}(t)+\hat{\sigma}_{\ln F}^{2}(t)}}\right)
\end{aligned}
$$

and reliability index $\beta(t)$ : 


$$
\begin{aligned}
\beta(t) & =\frac{\hat{\mu}_{\ln [F]}(t)-\hat{\mu}_{\ln F}(t)}{\sqrt{\hat{\sigma}_{\ln [F]}^{2}(t)+\hat{\sigma}_{\ln F}^{2}(t)}} \\
& =\frac{\left(\ln [F(0)]+\left(\mu_{[F]}-\frac{\sigma_{[F]}^{2}}{2}\right) t\right)-\left(\ln F(0)+\left(\mu_{F}-\frac{\sigma_{F}^{2}}{2}\right) \mathrm{t}\right)}{\sqrt{\left(\sigma_{[F]}^{2}+\sigma_{F}^{2}\right) t}}
\end{aligned}
$$

where, $\hat{\mu}_{\ln [F]}(t), \hat{\mu}_{\ln F}(t)$ are the mean of $\ln [F(t)], \ln F(t)$ respectively; $\hat{\sigma}_{\ln [F]}^{2}(t), \hat{\sigma}_{\ln F}^{2}(t)$ are the variance of $\ln [F(t)], \ln F(t)$ respectively.

Mean $\hat{\mu}_{\ln F}(t)$ and variance $\hat{\sigma}_{\ln F}^{2}(t)$ of the performance output $\ln F(t)$ can be calculated by the drift function $\mu_{F}$, the diffusion function $\sigma_{F}$. According to the multi-dimensional Ito Lemma (equation (4)), the drift function $\mu_{F}$, the diffusion function $\sigma_{F}$ of the performance output $F(t)$ can be expressed with the variables $x_{t}^{(i)}(i=1 \cdots \cdots n)$ of the system. The design variables $x_{t}^{(i)}$ all follows the Geometric Brownian Motion. If we design a system with the given reliability $\mathrm{R}(t)$ at specified time, the system performance output $\mathrm{F}(0)$ can be solved with equation (14).

If the system is designed to have the reliability $R(t)>R_{0}$ at time $\mathrm{t}=\mathrm{T}$, the reliability index $\beta(t)$ should meet $\beta(\mathrm{t}=\mathrm{T})>\Phi^{-1}\left(\mathrm{R}_{0}\right)$. For the reliability index $\beta(t)$ is a time-dependent continuous function, it is easy to calculate reliability index $\beta(t)$ over a time interval $[0, \mathrm{~T}]$.

\section{Estimation of Drift Rate $\lambda$ and Variance $\delta$ based on Time Series Data}

If the variable $x(t)$ follows the Geometric Brownian Motion, the drift rate $\lambda$ and diffusion rate $\delta$ of variable $x(t)$ can be obtained by related experiment data or operating data.

For the $\mathrm{n}+1$ observables value $\left\{x_{0}, x_{1}, \cdots \cdots, x_{\mathrm{n}}\right\}$ which sampling $x_{t}=x(t)$ at the time interval $\Delta=\mathrm{T}$ - $\mathrm{t}$, if $q_{\mathrm{t}}=\ln x_{t}-\ln x_{t-1}$, the mean and variance of the sampling data $q_{t}$ will be:

$$
\bar{q}=\frac{\sum_{t=1}^{n} q_{t}}{n}, s_{q}^{2}=\frac{1}{n-1} \sum_{t=1}^{n}\left(q_{t}-\bar{q}\right)^{2}
$$

When $\mathrm{n} \rightarrow \infty \quad$, it will have $\bar{q} \rightarrow \mathrm{E}(q), s_{q}^{2} \rightarrow \operatorname{Var}(q)$.

According to equation (10), it can yield the drift rate $\hat{\lambda}$ and diffusion rate $\hat{\delta}$ of $\ln x_{t}$ :

$$
\hat{\lambda}=\frac{\bar{q}}{\Delta}+\frac{\hat{\delta^{2}}}{2}, \hat{\delta}=\frac{s_{q}}{\sqrt{\Delta}}
$$

It can be seen in equation (16) that the drift rate and diffusion rate can be estimated from the mean and variance of the data series $q_{\mathrm{t}}$. For the multiple groups of sampling data, the estimated drift rate and diffusion rate can be achieved separately, and the average value of these estimated values will be regarded as the drift rate and diffusion rate of time-varying parameter. If we can get more sampling data of the variable $x(t)$, the estimated drift rate /diffusion rate will be more close to their real values.

\section{Examples of EBUD}

The system $S$ is composed of two sub-systems $S_{1}$ and $S_{2}$. The system $S$ operates properly if $S_{1}$ and $S_{2}$ working normally. The performance output of system $S_{1}$ is $S_{1}=x_{1} \cdot x_{2}$, and the performance output of system $S_{2}$ is $S_{2}=x_{2} / x_{3}$, where $x_{1}, x_{2}$ and $x_{3}$ are the design variables respectively. The value of allowable performance output $S_{1}$ and $S_{2}$ at the initial time is $\left[S_{1}(0)\right]=35,\left[S_{2}(0)\right]=35$. 
where the drift rate of allowable performance output $\left[S_{1}\right]$ and $\left[S_{2}\right]$ are $\lambda_{[S 1]}=-0.001, \lambda_{[S 2]}=-0.001$, the diffusion rate $\delta_{[S 1]}=0.005, \delta_{[S 2]}=0.005$.

For the $x_{1}, x_{2}$ and $x_{3}$ are the design variables of system $S$, the drift rate and diffusion rate of variables are given as following respectively:

$\mathrm{x} 1$ : drift rate $\lambda x_{1}=0.002$, diffusion rate $\delta x_{1}=0.01$;

$\mathrm{x} 2$ : drift rate $x_{2}=0.002$, diffusion rate $\delta x_{2}=0.01$;

$\mathrm{x} 3$ : drift rate入 $x_{3}=0.0002$, diffusion rateס $x_{3}=0.02$.

To design the system $S$ with the reliability $R(t)$ no less than $64.72 \%$ at time $\mathrm{t}=100$ hour, i.e. $\mathrm{P}\{S(100)$ $\leq[S(100)]\}$ no less than $64.72 \%$.

Solve:

The reliability $\mathrm{R}(\mathrm{t})$ of system $S$ is the product of two subsystem reliability $R_{1}(t)$ and $R_{2}(t)$.

Considering the subsystem $S_{1}$ has more complicated structure, its reliability $R_{1}$ can be lower, while the subsystem $S_{2}$ can have a little higher reliability $R_{2}$. We assigned the reliability $R_{1}(t)$ and $R_{2}(t)$ is about $R_{1}$ $(100)=65 \%, R_{2}(100)=99.6 \%$.

The drift function $\mu_{\mathrm{S} 1}$ and diffusion function $\sigma_{\mathrm{S} 1}$ of $\mathrm{S}_{1}$ can be given by Ito Lemma in equation (4):

$$
\left\{\begin{array}{l}
\mu_{S_{1}}=\frac{\partial S_{1}}{\partial x_{1}} \mu_{x_{1}}+\frac{\partial S_{1}}{\partial x_{2}} \mu_{x_{2}}=x_{2} \cdot \mu_{x_{1}}+x_{1} \cdot \mu_{x_{2}} \\
\sigma_{S_{1}}=\frac{\partial S_{1}}{\partial x_{1}} \sigma_{x_{1}}+\frac{\partial S_{1}}{\partial x_{2}} \sigma_{x_{2}}=x_{2} \cdot \sigma_{x_{1}}+x_{1} \cdot \sigma_{x_{2}}
\end{array}\right.
$$

Comparing the Ito process with the Geometric Brownian Motion of variables $x_{1}, x_{2}$, we can get: $\mu_{x 1}=x_{1}(t) \cdot \lambda x_{1}, \mu_{x 2}=x_{2}(t) \cdot \lambda x_{2}, \sigma_{x 1}=x_{1}(t) \cdot \delta x_{1}, \sigma_{x 2}=x_{2}(t) \cdot \delta x_{2}, \mu x_{3}=x_{3}(t) \cdot \delta x_{3}, \sigma x_{3}=x_{3}(t) \cdot \delta x_{3}$.

Using the expression of system $S_{1}$, it will have:

$$
\left\{\begin{array}{l}
\mu_{S_{1}}(t)=x_{2}(t) \cdot x_{1}(t) \cdot \lambda_{x_{1}}+x_{1}(t) \cdot x_{2}(t) \cdot \lambda_{x_{2}}=S_{1}(t) \cdot\left(\lambda_{x_{1}}+\lambda_{x_{2}}\right) \\
\sigma_{S_{1}}(t)=x_{2}(t) \cdot x_{1}(t) \cdot \delta_{x_{1}}+x_{1}(t) \cdot x_{2}(t) \cdot \delta_{x_{2}}=S_{1}(t) \cdot\left(\delta_{x_{1}}+\delta_{x_{2}}\right)
\end{array}\right.
$$

We use trial and error method to calculate the variables $x_{1}, x_{2}$ and $x_{3}$ at initial time. First, assume that $x_{1}(0)=5, x_{2}(0)=4$ and $x_{3}(0)=0.2$ thus $S_{1}(0)=20, S_{2}(0)=20$.

The system $S_{1}$ is an Ito process as equation (1) with drift rate $\lambda_{S 1}(t)$, diffusion rate $\delta_{S 1}(t)$ :

$$
\left\{\begin{array}{l}
\lambda_{S_{1}}=\lambda_{x_{1}}+\lambda_{x_{2}}=0.004 \\
\delta_{S_{1}}=\delta_{x_{1}}+\delta_{x_{2}}=0.02
\end{array}\right.
$$

Based on the knowledge in 2.3, it can lead to:

$$
\mathrm{d} \ln S_{1}=\left(\lambda_{S_{1}}-\frac{\delta_{S_{1}}^{2}}{2}\right) \mathrm{d} t+\delta_{S_{1}}^{2} \mathrm{~d} w_{t}
$$

It means that $\ln S_{1}$ will follow a normal distribution with mean $\hat{\mu}_{\ln S_{1}}=\ln S_{1}(0)+\left(\lambda_{S_{1}}-\frac{\delta_{S_{1}}^{2}}{2}\right) t$, and variance $\hat{\sigma}_{\operatorname{lnS}_{1}}(t)=\delta_{S_{1}}^{2} t$

So $\ln \left[S_{1}\right]$ will follow a normal distribution with mean $\hat{\mu}_{\ln \left[S_{1}\right]}(t)=\ln \left[S_{1}(0)\right]+\left(\lambda_{\left[S_{1}\right]}-\frac{\delta_{\left[S_{1}\right]}^{2}}{2}\right) t$, and variance $\hat{\sigma}_{\ln \left[\mathrm{S}_{1}\right]}(t)=\delta_{\left[S_{1}\right]}^{2} t$ at time $\mathrm{t}$.

The reliability of $R_{1}$ of the system $S_{1}$ at time instant $t=100 \mathrm{~h}$ will be: 


$$
\begin{aligned}
R_{1}(t=100) & =\Phi\left(\frac{\left[\ln \left[S_{1}(0)\right]+\left(\lambda_{\left[S_{1}\right]}-\frac{\delta_{\left[S_{1}\right]}^{2}}{2}\right) \times t\right]-\left[\ln S_{1}(0)+\left(\lambda_{S_{1}}-\frac{\delta_{S_{1}}^{2}}{2}\right) \times t\right]}{\sqrt{\left(\delta_{\left[S_{1}\right]}^{2}+\delta_{S_{1}}^{2}\right) \times t}}\right) \\
& =\Phi(0.3802) \approx 64.80 \%
\end{aligned}
$$

Same way to solve the system S2 ( Ito process):

$$
\begin{aligned}
R_{2}(t=100) & =\Phi\left(\frac{\left[\ln \left[S_{2}(0)\right]+\left(\lambda_{\left[S_{2}\right]}-\frac{\delta_{\left[S_{2}\right]}^{2}}{2}\right) \times t\right]-\left[\ln S_{2}(0)+\left(\lambda_{S_{2}}-\frac{\delta_{S_{2}}^{2}}{2}\right) \times t\right]}{\sqrt{\left(\delta_{\left[S_{2}\right]}^{2}+\delta_{S_{2}}^{2}\right) \times t}}\right) \\
& =\Phi(3.0032) \approx 99.87 \%
\end{aligned}
$$

The reliability $\mathrm{R}$ of the series system $\mathrm{S}$ can be verified that:

$$
R(t=100)=R_{1}(\mathrm{t}=100) \times R_{2}(\mathrm{t}=100)=0.6480 \times 0.9987 \approx 64.72 \%
$$

It means that the system $\mathrm{S}$ will have the reliability $\mathrm{R}$ at the time moment $\mathrm{t}=100$ hour. This designed system can meet the requirement of reliability.

\section{Conclusion}

The method of evolution-based uncertainty design (EBUD) for artificial systems is proposed in this paper considering the evolution and uncertain characteristics existing in the system. The performance output and allowable performance output of the system are defined as functions which can reflect the uncertainty and evolution characteristics of system state and requirement. In the view of EBUD, some notations such as uncertainty, reliability of the system are redefined and explained. The system in EBUD is described with the drift function and diffusion function, and the system reliability is expressed with the inequality relationship of the performance output and allowable performance output. The reliability of system in EBUD is dynamic variation and can be calculated at any time by using the observing data of the system variables.

In the system conceptual design period, the designer can know about the system reliability (or uncertainty) at any time. The EBUD supplies a method to forecast the trend of the system state. Two example demonstrates the EBUD is a universal method without focusing on the special system. The EBUD can have a widely application aspects, it can also be applied to tackle with such systems like city traffic system, city water supply and drainage system, gas system, nuclear system and logistic system.

\section{Acknowledgments}

The work is supported by the National Natural Science Foundation of China (Grant No. 51075029).

\section{References}

[1] Alessandro Birolini. Reliability Engineering - Theory and Practice, fifth ed., Springer-Verlag, 2007 
[2] Amandeep Singh, Zissimos P. Mourelatos, Jing Li, Design for lifecycle cost using time-dependent reliability, Mechanical Design,132(2010).

[3] G. Schall, M.H. Faber, and R. Rackwitz,The ergodicity assumption for sea states in the reliability estimation of offshore structures. Offshore Mechanics and Arctic Engineering,113(1991) 241-246.

[4] S.Engelund, R,Rackwitz, C.Lange, Approximations of first-passage times for differentiable processes based on high-order threshold crossing, Probabilistic Engineering Mechanics, 10(1995)53-600.

[5] C. Andrieu-Renaud, B. Sudret, M. Lemaire, The PHI2 method: a way to compute time-variant reliability, Reliability Engineering and System Safety, 84(2004) 75-86.

[6] Zhen Hu, Xiaoping Du. Time-dependent reliability analysis with joint upcrossing rates. Structural and Multidisciplinary Optimization, 48(2013) 893-907.

[7] Boqiang Shi, Yongye Yan, Huifang Fan, etc,Mechanical Design Method with Uncertain Evolution, Journal of University of Science and Technology Beijing, 30(2008) 1050-1054.

[8] Yongye Yan, Boqiang Shi,Time Dependent Reliability Analysis under Uncertainty, Journal of Xi'an Jiaotong University, 41(2007) 1303-1306. 